\title{
Article
}

\section{Individual differences in search and monitoring for color targets in dynamic visual displays}

Muhl-Richardson, Alexander, Godwin, Hayward J., Garner, Matthew, Hadwin, Julie A., Liversedge, Simon Paul and Donnelly, Nicholas

Available at https://clok.uclan.ac.uk/22323/

Muhl-Richardson, Alexander, Godwin, Hayward J., Garner, Matthew, Hadwin, Julie A., Liversedge, Simon Paul orcid iconORCID: 0000-0002-8579-8546 and Donnelly, Nicholas (2018) Individual differences in search and monitoring for color targets in dynamic visual displays. Journal of Experimental Psychology: Applied . ISSN 1076-898X

It is advisable to refer to the publisher's version if you intend to cite from the work. http://dx.doi.org/10.1037/xap0000155

For more information about UCLan's research in this area go to

http://www.uclan.ac.uk/researchgroups/ and search for <name of research Group>.

For information about Research generally at UCLan please go to http://www.uclan.ac.uk/research/

All outputs in CLoK are protected by Intellectual Property Rights law, including Copyright law. Copyright, IPR and Moral Rights for the works on this site are retained by the individual authors and/or other copyright owners. Terms and conditions for use of this material are defined in the policies page.

\section{CLoK}

Central Lancashire online Knowledge www.clok.uclan.ac.uk

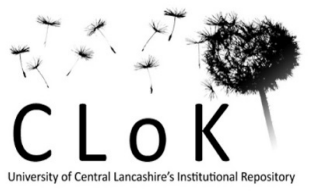


Individual Differences in Search and Monitoring for Color Targets in Dynamic Visual Displays

Alex Muhl-Richardson, Hayward J. Godwin, Matthew Garner, Julie A. Hadwin, Simon P. Liversedge and Nick Donnelly

University of Southampton

Author Note

Alex Muhl-Richardson, Hayward J. Godwin, Matthew Garner, Julie A. Hadwin, Simon P. Liversedge and Nick Donnelly, Psychology, University of Southampton, UK.

This research was conducted as part of the Defence Science and Technology Laboratory's National PhD scheme.

Correspondence regarding this article should be addressed to Alex Muhl-Richardson, Psychology, University of Southampton, Southampton, Hampshire, SO17 1BJ. Email: alex.muhl-richardson@soton.ac.uk 


\begin{abstract}
Many real-world tasks now involve monitoring visual representations of data that change dynamically over time. Monitoring dynamically changing displays for the onset of targets can be done in two ways: detecting targets directly, post-onset, or predicting their onset from the prior state of distractors. In the present study, participants' eye movements were measured as they monitored arrays of 108 colored squares whose colors changed systematically over time. Across three experiments, the data show that participants detected the onset of targets both directly and predictively. Experiments 1 and 2 showed that predictive detection was only possible when supported by sequential color changes that followed a scale ordered in color space. Experiment 3 included measures of individual differences in working memory capacity (WMC) and anxious affect and a manipulation of target prevalence in the search task. It found that predictive monitoring for targets, and decisions about target onsets, were influenced by interactions between individual differences in verbal and spatial WMC and intolerance of uncertainty, a characteristic that reflects worry about uncertain future events. The results have implications for the selection of individuals tasked with monitoring dynamic visual displays for target onsets.
\end{abstract}

\title{
Public Significance Statement
}

The present study shows that onset of color targets in dynamically changing displays can be predicted when changes follow an ordered color space. However, prediction can increase false alarm rate, especially for individuals with low verbal working memory capacity and high intolerance of uncertainty, a characteristic reflecting response to uncertain future events. Implications exist for color scale design when representing dynamically changing data and for personnel selection in display monitoring.

Keywords: visual search, eye movements, intolerance of uncertainty, working memory, anxiety 
Individual Differences in Search and Monitoring for Color Targets in Dynamic Visual

$$
\text { Displays }
$$

Monitoring electronic displays for the onset of targets is a key part of many real world activities and involves sustaining attention over a set of items whose state (or identity) can change (Warm, Finomore, Vidulich, \& Funke, 2015; Warm \& Parasuraman, 2008). There will be occasions when changes are minimal and all items remain as distractors requiring observers to take no action. There will also be occasions when some items switch from a distractor to a target state requiring a response to be made. The present set of experiments explores the psychological attributes that support the monitoring for the onset of a target color amongst an array of items that change color over the time course of a trial.

To provide a detailed real-world example of this type of scenario, consider the case where the colors of items in a display are coded to reflect the density of geological rock formations in a 3D volume (e.g. Donnelly, Cave, Welland, \& Menneer, 2006; see Figure 1). In this case, the density of sandstone might be coded as blue, limestone as white, and shale as red. A geologist might sequentially inspect 2D slices of this volume in the hope of finding red in the display, an indicator of hydrocarbon-rich shale deposits. This might be a simple task if displays contained only red, blue and white (i.e. detecting the presence of a red target amongst white and blue distractors). However, variations in density lead to subtle hue and luminance changes being presented and the heterogeneity of colors within the image makes the detection of targets amongst distractors more difficult (Duncan and Humphreys, 1991). The likelihood of overcoming these challenges might be enhanced if, over time, attention could be focused on likely targets rather than distributed across the whole display. For example, this would be so if the appearance of pink in the current slice being inspected might indicate the probable onset of red in a later slice. Of course, similar challenges occur in monitoring changing displays in less benign situations (see also Drew, Võ, \& Wolfe, 2013). 
There is very little visual search literature to inform our understanding of searching in displays of changing color items. Much of the evidence from dynamic search tasks involves stimuli that move or change in luminance (e.g. Kunar \& Watson, 2011, 2014). A recent study involving a dynamic orientation search task suggests that the additional load associated with organizing and updating representations of objects as they change state can significantly reduce search efficiency (Jardine \& Moore, 2015) but it is not clear how this might generalize to color search. Our starting point and working hypothesis is that initial monitoring for color targets uses a broad target template (Stroud, Menneer, Cave, \& Donnelly, 2012; Stroud, Menneer, Cave, Donnelly, \& Rayner, 2011) that allows prioritization of possible targets based on similarity to the target. If a distractor is prioritized it can be tested against a more specific target template when it changes color (e.g. changes from a pink to a deeper red).

The first objective, therefore, is to find and quantify evidence of predictive behavior in the search for color targets. This is addressed by recording basic behavioral data (hit rate, false alarm rate and response times) and eye movement data as participants search for the onset of either one or two color targets during a forty-second presentation of changing color items. The prediction is that distractors that are close to targets in color space will be prioritized for monitoring, and that this will be manifest in the eye movement record (i.e. increased fixations to distractors that neighbor targets in color space relative to other distractors).

The second objective is to test a specific hypothesis in relation to individual differences in predictive monitoring for color targets. In line with recently suggested guidelines for the study of individual differences in human factors research (Szalma, 2009), we have determined a set of relevant characteristics that might impact upon predictive monitoring. Prior research has shown that verbal and spatial working memory capacity (WMC) are associated with various factors important to search (Anderson, Mannan, Rees, Sumner, \& Kennard, 2008; Schwark, Sandry, Macdonald, \& Dolgov, 2012; Soto \& Humphreys, 2007), the ability to sustain attention over extended period of time (Caggiano \& Parasuraman, 2004), the 
maintenance of a task set (Kane, Bleckley, Conway, \& Engle, 2001; Mcvay \& Kane, 2012), maintaining attentional engagement (Sörqvist \& Marsh, 2015, p. 269), flexibility in the allocation of attention (Bleckley, Durso, Crutchfield, Engle, \& Khanna, 2003; Fukuda \& Vogel, 2011) and the inhibition of return to previously searched locations (Klein, 2000). More recently, it has been shown that visual WMC, attentional control and vigilance ability are particularly important predictors of performance in low prevalence visual search in particular (Peltier \& Becker, 2017).

Trait anxiety, and also state anxiety, are known to impact negatively on visual search (review by Richards, Benson, Donnelly, \& Hadwin, 2014) and reduce perceptual sensitivity (Pacheco-Unguetti, Acosta, Lupiáñez, Román, \& Derakshan, 2012). Theoretical frameworks like Attentional Control Theory (ACT) support the suggestion that the mechanism for this effect is that anxiety reduces the attentional resources (e.g. WMC) available for a given task (Derakshan \& Eysenck, 2009; Eysenck \& Derakshan, 2011; Eysenck, Derakshan, Santos, \& Calvo, 2007). This leads individuals with elevated anxiety to focus attention on internal (e.g., worrisome thoughts about performance) and/or external threat (e.g., potential negative evaluation from others). While ACT primarily considered trait anxiety, interference with attentional control has been demonstrated in individuals with high state anxiety (Derakshan, Smyth, \& Eysenck, 2009). The impact of elevated anxiety on task performance is particularly striking for individuals with low WMC (Eysenck \& Derakshan, 2011; Owens, Stevenson, Hadwin, \& Norgate, 2014). The present hypothesis is, therefore, that monitoring for the onset of color targets will be poor in individuals high in both trait and state anxiety and low in WMC.

Determining when distractors change to be targets is a decision that comes with a level of uncertainty. Intolerance of uncertainty (IU) is a characteristic that is linked to generalized anxiety (Birrell, Meares, Wilkinson, \& Freeston, 2011; Buhr \& Dugas, 2006) and individuals who report greater IU are more likely to take steps to avoid uncertainty or ambiguity (Birrell, Meares, Wilkinson, \& Freeston, 2011; Carleton, Norton, \& Asmundson, 2007). IU has been 
associated with attentional biases even after factors including anxiety, depression and distress were controlled for (Fergus, Bardeen, \& Wu, 2013; Fergus \& Carleton, 2016). When it is the case that responses resolve both targets and distractors by resetting their state, high IU is likely to impact specifically on response criterion. It is predicted that individuals with greater symptoms of IU will be more likely to respond simply to resolve the uncertainty surrounding the future state of target-similar distractors and will therefore demonstrate a greater likelihood of generating false alarms.

The present study utilized behavioral (hit rate, response time and false alarms) and eye movement measures (first fixations to targets, fixation durations, numbers of fixations and scanpath lengths) to examine target detection and monitoring in dynamically changing arrays of color stimuli. In addition to this, this study set out to test a specific hypothesis in relation to individual differences in order to determine who might perform well in this type of complex visual task. Color change dynamics were controlled such that stimuli changed according to sequential single-steps through an ordered set of 16 colors, to facilitate the prioritization and monitoring of target-similar distractors with a higher likelihood of becoming become targets. Experiments 1 and 2 included manipulations of these color change dynamics with a view to determining how and when forthcoming targets were predicted and monitored. Individual differences in WMC and anxious affect were considered in Experiment 3.

\section{Experiment 1}

The goal of Experiment 1 was to provide evidence that target detection in dynamically changing displays is supported by the selective prioritization and monitoring of targetpredictive distractors that are similar to targets. Participants searched for color targets within dynamically changing displays of colored squares while their eye movements were recorded. Across two experimental conditions the dynamics of color change were manipulated such that (1) stimuli changed according to sequential single-steps through an ordered set of 16 colors or 
(2) color changes were randomized. It was predicted that sequential single-step changes through a psychologically ordered color space would support a predictive search and monitoring strategy that would not be supported by randomized color changes and that such a strategy would improve the speed and accuracy of target detection. The difference between conditions should be evident in the eye movements made to targets and distractors whose presence might indicate an emerging target. Eye movement recordings were used to determine when targets and forthcoming targets were first fixated as a function of the number of color steps from the target color. By definition, if targets were first fixated at the target color, this would not indicate predictive monitoring. However, if forthcoming targets were first fixated as target-predictive distractors, operationalized here as one or two steps from the target color, then this would be consistent with predictive monitoring. In the condition of randomized color changes, a target onset could follow any of the non-target colors. This meant that no distractor was predictive of target onsets in the randomized color change condition. In addition to reporting eye movement data, hit and false alarm rates and response times (RTs) are also reported.

\section{Method}

Design. The independent variable was the type of change (sequential or random) that occurred within the displays and participants completed a block of each condition in counterbalanced order. There were multiple dependent variables. Performance across each experimental condition was recorded in terms of RT, hit rate and false alarm rate. Eye movement recordings were used to determine the proportion of first fixations on targets and forthcoming targets 1 and 2 color steps from the target color.

Participants. Sixteen undergraduate and postgraduate students ( 13 females; $M_{\text {age }}=24.8$ years; $S D=5.5$; age range: $19-41$ years) participated in the study for course credits or compensation of $£ 7.50$. All participants had normal visual acuity (at least 1.0 decimal VA at 
$70 \mathrm{~cm}$ ), tested using the Freiburg Visual Acuity Test (Bach, 1996), and normal color vision, tested using the City University Color Vision Test 3rd Edition (Fletcher, 1998). All experiments in the current paper were approved by the ethics committee of the School of Psychology at the University of Southampton and by the Ministry of Defence Research Ethics Committee. This sample size was chosen to ensure that each of the 16 colors was assigned to at least one participant as a target.

Apparatus. Stimuli were displayed on a 21-inch CRT monitor operating at a resolution of 1,024 x 768 and a refresh rate of $120 \mathrm{~Hz}$ connected to an SR Research EyeLink 1000 eyetracker operating at a sampling rate of $1000 \mathrm{~Hz}$. A nine-point calibration procedure was used and calibration was accepted only when none of the points had an error of more than $0.5^{\circ}$ of visual angle. Participants were seated $70 \mathrm{~cm}$ from the display, the display was viewed binocularly, although only the right eye was tracked, and a chin rest was used to keep participants' heads stable during the experiment. The experiment was programmed using SR Research Experiment Builder, with additional custom code written in Python.

Stimulus spatial properties. Visual angles were calculated from the center of the display (values vary marginally for more eccentric locations). Individual colored squares (items), were $0.57^{\circ} \times 0.57^{\circ}$, and displayed in irregular $12 \times 9$ arrays. Items never abutted, but were randomly 'jittered' within the constraints of an invisible grid, such that each stimulus appeared within an area of $2.15^{\circ}$ by $2.15^{\circ}$, which was used to code fixations. The maximum size of the whole array of color stimuli was $24.78^{\circ} \times 18.36^{\circ}$ and 108 items were always present. Figure 2 shows a sample display.

Stimulus color properties. Stimuli were colored using a 16-item color scale (see Menneer et al., 2007). For each participant, one color was the target (T) and 15 colors were distractors (D). Of the 108 colored squares, 96 squares were always at least 3 steps away from being a target and these were considered background distractors. There were 12 squares that were allowed to display colors within 2 color steps of the target. The locations of these 12 
squares were varied across trials and, while unknown to participants, these were never located at the edge of the array or immediately adjacent another of these 12 squares. The target color was varied and counterbalanced between participants, such that each color was the target for one participant (Figure 3 shows the full color scale).

Stimulus dynamic properties. All stimuli changed color over the course of each trial. Stimulus arrays were updated with a variable refresh rate, this resulted in displays that refreshed at a mean rate of once every $107 \mathrm{~ms}(S D=100)$ and the mean rate of color change for each item was a change every $336 \mathrm{~ms}(S D=342)$. Background distractors in both conditions could change between the 11 colors that were at least 3 steps from the target color. When changing color, items could change to be closer to, or further from, the target color. For background distractors, these two possibilities were equally likely, other than when three steps from the target color. At certain time points during trials, the 12 squares that could display colors within 2 steps of the target were allowed to change across the full range of 16 colors (or 15 colors if they were not to become a target). This was the case in both the ordered and randomized conditions. As there were up to two targets per trial, at least 10 of these 12 stimuli reached a color one step from the target, but never achieved a target state. The effect of these conditions was to allow stimuli in the ordered condition to systematically approach the target color, allowing the color of distractors to be used as a predictive cue to the onset of targets. Color could not be used as a cue to predict the onset of targets in the randomized condition. Each of these 12 items was given a number of key properties, all independently and pseudo-randomly generated within Python: (1) a time at which they were allowed to move within two colors of the target; (2) a time after which they will become a target or a distractor color one step from the target; and (3) a duration for which they remain present as target or a distractor one step from the target before reverting to background distractor (if no response was made). These values corresponded to the first item starting to move towards a target state after an average of $3,550 \mathrm{~ms}(S D=1,915)$, the first target onset after an average of $14,886 \mathrm{~ms}(S D=$ 
$1,569)$, the second target onset (where applicable) after an average of $18,845 \mathrm{~ms}(S D=2,480)$. This also resulted in an average duration of targets, if no response was made, of 2,549 $\mathrm{ms}$ ( $S D$ $=1,768)$. The parameters that controlled stimulus behavior were selected after piloting to allow for: (1) the possibility of target detection and prediction without excessive difficulty; (2) to ensure that no item could start to approach a target state immediately following the start, or immediately before the end, of a trial; and (3) to allow for inter-trial variation. Responding target-present (irrespective of whether it was a hit or a false alarm) also caused it to reset to a background distractor. While up to two targets might be presented over the course of a trial, only one target could be present at any given time. While color changes were more salient in the randomized than ordered condition, the relative salience of changes from distractor-totarget and distractor-to-distractor were matched across conditions. A video of a sample trial is included as online supplementary material.

Procedure. Participants were first tested for normal visual acuity and color vision. Participants were shown a static sample of the search display (as in Figure 2). They were instructed to search for a target square defined by a particular color, which was shown before every trial, and that responses were to be made as quickly as possible by clicking the mouse cursor on the target. Participants were told that trials might contain no targets, a single target or more than one target, but that only one target would appear at a time. Trials began with a $1 \mathrm{~s}$ reminder of the target color, followed by a $1 \mathrm{~s}$ fixation point and each trial lasted $40 \mathrm{~s}$ (see Figure 4 for the trial sequence). Audio feedback indicating either 'correct' or 'incorrect' was given for every response. Participants were given a self-paced break halfway through each block of 40 trials. Participants completed two blocks of trials (one each for the ordered and randomized conditions). In the ordered condition, all color changes occurred in single sequential steps through the 16 possible colors and stimuli never changed in color by more than a single step. In the randomized condition items were controlled in the same way but any color change could be between 1 and 8 steps. In both blocks, 10 out of 40 trials had a single target 
present and 10 out of 40 trials had two targets present. A practice block of three trials, with sequential color change dynamics, was given before beginning the first block.

\section{Results}

RT exclusion criteria were set a priori at above 10,000 ms and below $200 \mathrm{~ms}$ from target onset but no responses were outside of these limits. RTs were log transformed before being analyzed and untransformed means are also reported. In the eye movement data, fixations were excluded from duration analysis if they were longer than 1,200 ms, shorter than $80 \mathrm{~ms}$ in duration or if they corresponded with a manual response (3.98\% of all fixations). Proportional data were arcsine square root transformed before being analyzed and untransformed means are also reported. Hit rate was defined as the proportion of targets across all trials that were responded to correctly. False alarm rate was defined as the proportion of all responses that were made to non-target stimuli ${ }^{1}$.

Behavioral performance. For descriptive statistics of the hit rate, RT and false alarm rate see Table 1 . Contrary to predictions, performance did not differ between ordered and randomized conditions in terms of hit rate, $t(15)=0.96, p=0.35$, RT, $t(15)=0.65, p=0.53$, or false alarm rate, $t(15)=0.20, p=0.84$.

Target prediction. To examine target prediction, first fixations to targets and forthcoming targets were analyzed in the ordered condition. In the randomized condition first fixations to targets and forthcoming targets in a distractor state one or two steps from becoming a target were analyzed (see Figure 5). Only first fixations to forthcoming targets that went on to receive a response are included. First fixations give a measure of the state of items when they

${ }^{1}$ We do not calculate signal detection theory (SDT) measures of sensitivity or response criterion due to the non-standard calculation of false alarm rate. In the present case, the false alarm rate does not equal one minus the correct rejection rate (FAR $\neq 1-\mathrm{CRR})$, but instead equals one minus the hit rate $(\mathrm{FAR}=1-\mathrm{HR})$. 
initially attracted attention. These data were analyzed in a 2 (display type: ordered and randomized) x 3 (color step: $\mathrm{T}, \mathrm{T}+/-1, \mathrm{~T}+/-2$ ) repeated-measures ANOVA.

As a difference in the ability to predict target onset was expected between display types, it is the interaction between color steps and display type that addresses our first research question. Color step and display type interacted, $F(2,30)=26.62, p<.001, \eta^{2}{ }_{G}=0.37$. Pairwise comparisons of display type at each number of color steps from the target (Bonferroni-corrected $\alpha=.017$ ) revealed a significant difference for fixations to distractors at one step, $t(15)=8.19, p<.001$, but no difference between display types for fixations to targets, $t(15)=1.81, p=.09$, or distractors at two steps, $t(15)=2.33, p=.03$. The analysis revealed evidence of targets being first fixated when targets in both ordered and randomized displays and of predictive fixations (fixations to forthcoming targets prior to onset of the target color) in ordered displays only. The ability to make predictive fixations to forthcoming targets, in ordered displays, makes these distractors predictive of targets and, henceforth, we refer to these as target-predictive distractors (TPDs).

In addition, analysis of fixations and refixations (an index of monitoring over time) to targets and TPDs in the ordered condition only showed a mean of $2.87(S D=2.14)$ total fixations to items first fixated as targets, and $2.26(S D=1.55)$ and $1.46(S D=0.51)$ total fixations to TPDs first fixated one and two steps respectively from the target. Fixations to background distractors in the ordered condition accounted for an average of $31.52 \%(S D=$ 7.25) of all fixations made across all participants and each background distractor was fixated on average 0.88 times $(S D=1.34)$.

We explored the effect of predicting forthcoming targets with respect to hit rate, RT and false alarm rate for forthcoming targets first fixated as TPDs at $\mathrm{T}+/-1$ (these data are shown in Figure 6 for all experiments). There were too few data to allow meaningful analysis of predicting forthcoming targets at $\mathrm{T}+/-2$ steps. This analysis revealed that predicting 
upcoming targets: (1) reduced the hit rate for targets first fixated as TPDs one step from becoming a target $(M=0.81, S D=0.16)$ relative to first fixating targets $(M=0.93, S D=0.07)$, $t(15)=2.71, p=.02 ;(2)$ did not speed RT for those items first fixated as a TPD one step from becoming a target $(M=2,539 \mathrm{~ms}, S D=659)$ compared to when first fixating a target itself $(M$ $=2,740 \mathrm{~ms}, S D=557), t(15)=1.29, p=.22$; and (3) led to a false alarm rate for targets first fixated as TPDs one step from becoming targets $(M=0.27, S D=0.30)$ that was significantly greater than zero, $t(15)=3.64, p=.002$.

\section{Discussion}

Experiment 1 found no evidence of the predicted benefit to hit rate or RT of presenting colors in an ordered versus a randomized sequence. These results suggest that the salience of color change in ordered and randomized conditions did not impact overall performance.

However, Experiment 1 demonstrated that single step color changes enabled the prediction of forthcoming targets via the fixation of TPDs close to targets in color space in the ordered condition. In the ordered condition, first fixations were as likely to be made to distractors one step from the target color as to targets themselves. While it was predicted that first fixating TPDs one step from being a target would benefit target detection overall, results were consistent with reduced target sensitivity. For those items first fixated as TPDs, hit rates were reduced relative to when items were first fixated as targets and false alarms were significantly greater than zero.

The results were consistent with an account whereby participants used a broad target template to locate targets or target-similar stimuli one step either side of the target color, though there was no evidence that using a broadened template actually improved task performance overall. The reason for this is simple. False alarms occurred on roughly one quarter of occasions when fixating distractors that were close in color to targets. Although participants were monitoring distractors that could soon become targets, the mechanism of 
prediction was problematic. In some cases, this led to false alarms, where premature responses were made to TPDs before they could become targets. In other cases, this led to misses, where TPDs were fixated but discounted as possible targets and received no response following the onset of the target color. In the context of equivalent overall performance in the randomized and ordered conditions, the existence of these sources of error in relation must imply that more potential targets were being identified in the ordered than randomized condition.

Evidence of uncertainty in relation to detecting targets was found in the analysis of refixations. Refixations were more common when items were first fixated when in the target state and declined monotonically with distance from the target. First fixating a target when in a target state appeared to lead to checking and, in contrast, first fixating a target as a TPD led to sustained attention, reduced checking and an increased false alarm rate. In other words, the tendency to false alarm, in the context of the resetting of color state following responses, contributed to the failure to find evidence of an overall benefit on hit rate of monitoring for target onsets in Experiment 1.

Experiment 2 explored a potential alternative account of the effects of order color change dynamics found in Experiment 1. It is possible that predictive monitoring for targets in dynamically changing color displays does not rely on use of an ordered color space but rather on any predictable pattern of change between color, for example, if a red distractor always preceded a blue target then this association might be learned and influence search. In the real-world, color scales used in a variety of imaging and mapping tasks can be defined by the user, for example, in choropleth maps. This might result in arbitrary color coding that follows a fixed pattern, but does not involve a psychologically ordered color space.

\section{Experiment 2}

Participants completed the same task as in Experiment 1, with a change in the manipulation of display ordering. The block of the task with randomized color changes was 
replaced with a block where color changes occurred in a predictable pattern but through a shuffled color space and target-predictive distractors were not target-similar colors. As in Experiment 1, it was predicted that color changes according to an ordered color space would support a predictive search strategy. However, when stimuli changed according to a shuffled color space, it was predicted that the use of a predictive strategy would be very limited due to the requirement to learn color associations. As before, the extent to which participants are able to engage in predictive target detection will be reflected in the proportion of first fixations to TPDs, with more first fixations to TPDs indicating greater predictive detection.

\section{Method}

The method, including the design, apparatus, stimuli and procedure, was the same as for Experiment 1 except for the differences below.

Participants. 16 undergraduate and postgraduate students ( 15 female; $M_{\text {age }}=21.6$ years; $S D=5.7$; age range: $18-38$ years) participated in Experiment 2. This sample size was chosen to ensure that each of the 16 colors was assigned to at least one participant as a target.

Stimulus dynamic properties. Display dynamics were manipulated by generating a pseudo-randomly shuffled (constrained to maintain discontinuity in color space) color change sequence for each participant, using the same 16 colors as in Experiment 1 (see Figure 2 for an example). While color changes were more salient in the shuffled than ordered condition, the relative salience of changes from distractor-to-target and distractor-to-distractor were matched across conditions. Each participant completed a block of the task under these conditions and a block under the ordered conditions described in Experiment 1.

\section{Results}

The data were analyzed as in Experiment 1 and, using the same exclusion criteria, $0.31 \%$ of all RTs and $3.52 \%$ of all fixations were removed before behavioral and eye movement analyses. 
Behavioral performance. Hit rate was higher, $t(15)=8.13, p<.001$, and RT was faster, $t(15)=7.87, p<.001$, in the ordered condition compared to the shuffled. False alarm rate did not differ significantly between conditions, $t(15)=1.13, p=0.28$ (see Table 1 ).

Target prediction. With respect to first fixations to targets, the eye movement data revealed a significant main effect of color step, $F(2,30)=287, p<.001, \eta^{2}{ }_{G}=0.84$. As in Experiment 1, where a difference in target prediction was expected between display types, it is the interaction between color step and display type that is of interest. Color step and display type interacted, $F(2,30)=36.06, p<.001, \eta^{2}{ }_{G}=0.62$, (see Figure 5). Pairwise comparisons (Bonferroni-corrected $\alpha=.017$ ) of display type at each color step revealed that a greater proportion of first fixations were made to targets in shuffled compared to ordered displays, $t(15)=5.73, p<.001$. A greater proportion of first fixations were made to distractors one step from the target in ordered than shuffled displays, $t(15)=7.10, p<.001$ (see Figure 5) and there was a non-significant difference in first fixations made to distractors two steps from the target color, $t(15)=1.99, p=.06$. The analysis shows a much greater reliance on first fixating targets in the shuffled than ordered condition and replicates the results found in Experiment 1 for the ordered condition, in particular, fixations were made to TPDs in the ordered condition.

Analysis of fixations and refixations to targets and TPDs in the ordered condition showed a mean of $2.71(S D=1.94)$ total fixations to targets, and $2.04(S D=1.35)$ and 1.58 $(S D=1.17)$ total fixations to TPDs one and two steps from the target respectively. Fixations to background distractors in the ordered condition accounted for an average of $31.03 \%(S D=$ 6.60) of all fixations made across all participants and each background distractor was fixated on average 0.87 times $(S D=1.26)$.

The same approach was taken as in Experiment 1 with regard to examining the influence of predicting forthcoming targets on behavioral measures. Experiment 2 confirmed the pattern of behavioral data reported in Experiment 1. In the ordered condition of Experiment 
2: (1) hit rate was marginally lower when first fixating a TPD one step from becoming a target $(M=0.86 S D=0.16)$ than when first fixating the target itself $(M=0.94 S D=0.07)$, but this effect did not reach significance, $t(15)=1.91, p=.08$ (despite the difference in SD, a BrownForsythe test confirmed equality of variance in these data); (2) there was no difference in RTs to targets first fixated as TPDs one step from becoming a target $(M=2,677 \mathrm{~ms}, S D=961)$ and those first fixated as targets $(M=2,500 \mathrm{~ms}, S D=553), t(15)=0.70, p=.49$; and (3) the false alarm rate for targets first fixated one step away from the target color $(M=0.29, S D=0.30)$ was significantly greater than zero, $t(15)=3.86, p=.002$.

\section{Discussion}

The results from the ordered condition of Experiment 2 replicate those from Experiment 1. Overall performance was similar across the two studies. Target detection occurred following direct fixation of targets, as well as the monitoring of target-predictive distractors. The monitoring of target-predictive distractors led to false-alarms and a marginally reduced hit rate. The number of fixations to targets also reduced monotonically across targets and TPDs with distance from target. The data are consistent with an error prone predictive monitoring process for upcoming targets.

With respect to displays in the shuffled condition, participants found target detection very challenging and overall performance was less accurate and slower than in the ordered condition. This might be partially accounted for by the increased salience of color changes in the shuffled than ordered condition, although this is unlikely given the results of Experiment 1 and the high salience of color changes within the randomized condition. The striking difference between performance in the randomized condition of Experiment 1 and the shuffled condition of Experiment 2 is important. This result suggests that participants were able to ignore randomized color changes in their search for the onset of a color target, however, they found it very difficult to ignore systematic color changes to distractors where changes were not drawn 
from a psychologically ordered color space. In other words, systematic changes made outside of a psychologically ordered color space interfered with the detection of targets.

There was no evidence of predictive target detection in the shuffled condition, as target present responses almost exclusively followed first fixations to targets, and participants showed much longer RTs to targets. Together these data suggest that it was hard for participants to learn the color associations necessary to make use of TPDs in this condition. Whether participants noticed the shuffled color sequences was not recorded as part of the formal debriefing, however, no participants indicated that they were aware of a pattern of color changes in the shuffled condition. Informally, many participants indicated that they found target detection particularly difficult in the shuffled condition, which was consistent with behavioral performance.

Together, it is suggested that Experiments 1 and 2 are consistent with participants using a broad target template to guide search to facilitate predictive monitoring for targets stimuli (Hout \& Goldinger, 2015; Stroud et al., 2012, 2011). This is only possible when stimuli change color according to an ordered color scale and the template is both broad and narrow enough, to guide search to a color range that includes the target and TPDs. Importantly, for accurate target detection the use of this broad template must be followed by a separate perceptual decision, based on a more specific template. This second decision involves a more precise target specification but the data suggest that this may be a challenging and error prone process, as this would account for the lowered hit and raised false alarm rates found to targets first fixated as target-predictive distractors relative to those first fixated as targets.

Determining those individuals likely to make such errors in predictive monitoring tasks is a question of significant theoretical and practical significance. Experiment 3 aimed to extend the findings of Experiments 1 and 2 to investigate individual differences that might be associated with errors in this type of monitoring task. 


\section{Experiment 3}

Experiments 1 and 2 found evidence that participants engaged in predictive monitoring for targets in arrays of changing color items. One suggested mechanism that would support such predictive monitoring is the use of a broad target template to first identify items broadly similar to targets, followed by a decision made with respect to a more specific template. Engaging in predictive monitoring in changing displays may be dependent on a number of individual factors. Experiment 3 examined whether predictive monitoring is associated with individual differences in WMC and self-reported trait and state anxiety and IU.

Only ordered displays were used in Experiment 3 and, to increase task difficulty, a manipulation of target prevalence was added. To ensure that the effect of individual differences could be assessed at both low and high prevalence levels, target prevalence was manipulated within participants. This introduces the possibility of carry-over effects between blocks of different prevalence levels as individuals adjust their response criterion to meet the changed task parameters (Vickers \& Leary, 1983). However, the feedback provided in the present task should speed this adjustment (Wolfe et al., 2007) and order counterbalancing ensured that any remaining carry-over effects are distributed equally between groups. Consistent with established effects of low prevalence in visual search (e.g. Godwin, Menneer, Riggs, Cave, \& Donnelly, 2014), it was hypothesized that reduced target prevalence would lower the number of first fixations to targets and the hit rate and increase RTs and the false alarm rate. Moreover, as low prevalence increases uncertainty, it was expected that prevalence would interact with anxiety and IU, such that the effects of low prevalence would be most evidence in individuals with symptoms of anxiety and IU (and low WMC). Additional eye movement measures of the number of fixations, fixation duration and scanpath length are included to further determine the effects of these individual differences upon monitoring behavior. 


\section{Method}

The method was the same as for Experiment 1 except for the differences noted below.

Design. Target prevalence had two levels, high (66\%) and low (5.6\%), and was manipulated within participants, such that all participants completed one block at each level of prevalence and these were order counterbalanced.

Participants. 33 undergraduate and postgraduate students participated in the study (23 female; $M_{\text {age }}=23.9$ years; $S D=5.7$; age range: $18-36$ years). Participants completed the study for course credit or were compensated for their time at a rate of $£ 6$ per hour. This sample size was chosen to ensure that each of the 16 colors was assigned to at least two participants as a target.

Working memory capacity. WMC was measured using spatial and verbal 3-back working memory tasks which were identical in appearance and means of response (Shackman et al., 2006). The spatial task required remembering locations and the verbal task required remembering a letter and, in each case, there were six possible locations or letters that could appear. Trials lasted $500 \mathrm{~ms}$ and there was a 2,500 ms interval between trials. The task was continuous and every trial required a response to indicate either a match or non-match with the stimulus presented three trials previously. No feedback was given and the task proceeded automatically if no response was made.

Trait and state anxiety. The Spielberger State-Trait Anxiety Inventory Form Y (STAI; Spielberger, Gorsuch, Lushene, Vagg, \& Jacobs, 1983) consists of two 20-item self-report measures of anxiety for adults. The two scales, one measuring state anxiety and the other trait anxiety, provide separate scores and each response is made on a four-point scale, generating a total score from 20 to 80 for each. In the current study the reliability of both scales was acceptable $(\alpha>$.77). Approximately $30.3 \%$ of the present sample reported elevated scores (i.e., total scores $>40$ ) on the STAI trait scale (Lam, Michalak, \& Swinson, 2005). 
Intolerance of uncertainty. The Intolerance of Uncertainty Short Form (IUS-12; Carleton et al., 2007) is a 12-item self-report measure that assesses reactions to uncertainty, ambiguous situations and future events. It consists of two factors, the first, prospective IU, relates to worry regarding future events and is assessed by 7 items, for example, "I can't stand being taken by surprise." The second, inhibitory IU, relates to the extent to which uncertainty might inhibit action or experience and is assessed by 5 items assessing, for example, "when it's time to act, uncertainty paralyses me." Each response is made on a five-point scale which ranges from 1 "not at all characteristic of me" to 5 "entirely characteristic of me" and this results in a total score between 12 and 60 . In the current study the reliability of the scale was excellent $(\alpha>.92)$.

Apparatus and materials. The STAI and the IUS-12 were administered using paper questionnaires and the working memory tests were administered on the same computer as the main experiment (see Experiment 1).

Procedure. Participants completed the STAI and IUS-12, followed by the spatial and verbal 3-back working memory tasks. The state component of the STAI was completed a further three times: immediately before the search task, between blocks of the search task and at the end of the session. Participants completed two 72-trial blocks of the search task, one at high target prevalence and one at low prevalence, and were given no instructions or information regarding target prevalence levels. In low prevalence blocks, 2 out of 72 trials had a single target present and 2 out of 72 trials had two targets present, such that 4 out of 72 trials (5.6\%) had a target present. In high prevalence blocks, 24 out of 72 trials had a single target present and 24 out of 72 trials had two targets present, such that 48 out of 72 trials $(66 \%)$ had a target present. 


\section{Results}

The 3-back task data from three participants was incomplete and was not included analysis of WMC. Preliminary analysis of eye movements from the low prevalence condition showed that, given the low number of targets, some participants did not fixate targets as TPDs and therefore did not contribute to analysis of these data. Two approaches were taken in data analysis, with the relevant data being analyzed as in Experiments 1 and 2 and the individual differences data being analyzed using linear mixed models (LMMs).

Behavioral responses. The behavioral data, including hit rate, $\mathrm{RT}$, false alarm rate and first fixations to Ts/TPDs, were analysed as in Experiments 1 and 2. The only difference being that the 'display type' factor was replaced with 'prevalence' (with two levels, high and low).

Hit rate did not differ significantly between prevalence levels, $t(32)=0.20, p=.84$. RT was faster, $t(15)=2.57, p=.02$, and false alarm rate was lower, $t(15)=6.51, p<.001$, at high target prevalence compared to low (see Table 1).

Target prediction. With respect to predictive monitoring, there was a significant main effect of color step, $F(2,64)=60.91, p<.001, \eta^{2}{ }^{2}=0.54$, demonstrating that first fixations to TPDs one step from becoming targets were higher than TPDs two steps from becoming targets (see Figure 7). In addition, there was a significant interaction between color step and target prevalence, $\left.F(2,64)=3.99, p=0.02, \eta^{2} G=0.04\right)$. In the low prevalence conditions first fixations fell monotonically from targets through to distractors two steps from becoming targets. In the high prevalence conditions, numerically more targets were first fixated when TPDs one step from becoming targets then when targets themselves. However, pairwise comparisons (Bonferroni-corrected $\alpha=.017$ ) across prevalence levels revealed no significant differences in first fixations to targets, $t(32)=1.73, p=0.09$, or TPDs one step from becoming a target, $t(32)=1.21, p=0.24$, and so the interaction between when first fixations were made to targets and prevalence will not be considered any further. 
Analysis of fixations and refixations to targets and TPDs at high prevalence showed a mean of $2.53(S D=0.43)$ total fixations to targets, and $2.01(S D=0.28)$ and $1.63(S D=0.42)$ total fixations to TPDs one and two steps from the target respectively. At low prevalence, there was a mean of $2.73(S D=1.11)$ total fixations to targets, and $1.92(S D=0.68)$ and $1.64(S D=$ 0.92) total fixations to TPDs one and two steps from the target respectively. Fixations to background distractors across both prevalence conditions accounted for an average of $31.48 \%$ $(S D=7.27)$ of all fixations made across all participants and background distractors that received fixations were fixated on average 0.82 times $(S D=1.31)$.

Next the effects of predictive monitoring on hit rate, RTs and false alarm rate, as in Experiments 1 and 2, are reported, but with a distinction between high and low prevalence conditions. The same approach was taken as in Experiment 1 with regard to examining the influence of predicting forthcoming targets on behavioral measures. At low prevalence: (1) hit rate was not significantly lower when first fixating a TPD one step from becoming a target $(M$ $=0.86, S D=0.28)$ than when first fixating the target itself $(M=0.94, S D=0.15), t(28)=1.27$, $p=.22$; (2) RT was not significantly different when first fixating a TPD one step from becoming a target $(M=3,124 \mathrm{~ms}, S D=2,505)$ than when first fixating the target itself $(M=$ $3,138 \mathrm{~ms}, S D=1,426), t(26)=0.81, p=.42$; and (3) the false alarm rate for targets first fixated as TPDs one step from becoming a target $(M=0.59, S D=0.40)$ was significantly greater than zero, $t(32)=8.56, p<.001$.

At high prevalence: (1) hit rate was significantly lower when first fixating a TPD one step from becoming a target $(M=0.88, S D=0.11)$ than when first fixating the target itself $(M$ $=0.95, S D=0.06), t(32)=3.97, p<.001 ;(2) \mathrm{RT}$ was not significantly lower when first fixating a TPD one step from becoming a target $(M=2,604 \mathrm{~ms}, S D=622)$ than when first fixating the target itself $(M=2,692 \mathrm{~ms}, S D=614), t(32)=0.88, p=.38$; and (3) the false alarm rate to targets first fixated as TPDs one step from becoming a target $(M=0.18, S D=0.22)$ was 
significantly greater than zero, $t(32)=4.76, p<.001$. Overall, these experimental data from Experiment 3 confirm the findings from Experiments 1 and 2.

Individual differences. LMM analyses were performed in R (v3.0.3) using the lme4 package (v1.1-7; Bates, Mächler, Bolker, \& Walker, 2014). These models allow analysis of these data and the interactions among the variables as continuous variables and, because they do not rely upon aggregated means, are more robust to missing data, outliers and unbalanced cell counts. All models included participant as a random factor and, in all cases, model fitting began with a model containing the full set of two-way interactions and iterated through different variants until reaching the best-fitting model (any models that failed to converge were excluded). The effects of individual differences in spatial WMC, verbal WMC, trait anxiety, state anxiety and IU were considered in relation to a number of behavioral and eye movement measures. RT, fixation duration, number of fixations per trial and scanpath length and an index of the extent predictive fixations to TPDs were all analyzed using standard LMMs. Hit rate and false alarm rate were analyzed using binomial generalized linear mixed models (GLMMs). In the case of the analysis of hit rate, every target was entered into the model as a binary value indicating whether or not that target received a hit. Similarly, for the analysis of false alarm rate, every response was entered into the model as a binary value indicating whether or not that response was a false alarm. In addition to the robustness these models, this approach offers increased statistical power and sensitivity to potentially subtle effects.

The mean overall score on the STAI trait scale was $38.21(S D=9.50)$ and the mean overall score collapsed across all time points on the STAI state scale was $34.42(S D=7.63)$. The mean overall score on the IUS-12 was $28.72(S D=9.96)$. STAI trait and state scores were positively correlated $(r=0.64)$. IUS-12 scores were positively correlated with STAI trait $(r=$ $0.51)$ and state scores $(r=0.56)$.

Inspection of Table 2 reveals a number of important effects and interactions. First, IU is positively associated with false alarm rate and also interacts with verbal WMC (see Figure 8). 
The overarching explanation is that low verbal WMC paired with increased symptoms of IU changes the decision threshold employed by participants to respond 'target-present' such that the false alarm rate increases. This effect was more pronounced at low, compared to high, target prevalence and there was also a corresponding effect in the eye movement record in terms of the number of fixations made, with low verbal WMC paired with increased IU symptoms being associated with an increased number of fixations. Second, spatial WMC and IU interact with respect to the extent predictive first fixations (see Figure 8). When spatial WMC and IU are low then there is a reduced likelihood of first fixating targets as TPDs. Third, some effects are only evident in conditions of low or high target prevalence. Shorter scanpaths and lower numbers of fixations were found at low prevalence relative to high and IU was positively associated with the number of fixations at low but not high prevalence. It was also only in conditions of high target prevalence that trait anxiety was positive related to scanpath length.

\section{Discussion}

Experiment 3 confirmed the basic findings from the ordered conditions in Experiments 1 and 2. Participants monitored target-predictive distractors and this was the case even when targets occurred very rarely. In addition, low target prevalence led to an overall increase in false alarm rate, i.e. incorrectly classifying distractors as targets, slowed response times (although this effect was only evident when comparing means) and individuals with increased IU symptoms were particularly susceptible to this effect. Low target prevalence also led to an overall shift in search strategy evident by reduced scanpath lengths and the number of fixations made.

In terms of simple behavioral measures, individual differences predicted the false alarm rate, however, they also predicted a range of eye movement measures. Specifically, there was an influence of IU, across false alarm rate and eye movement measures, in interaction with 
either spatial or verbal WMC. A simple characterization of the pattern of results is that IU is problematic for predictive search when associated with low spatial or verbal working memory. A consequence of increased IU symptoms combined with low verbal working memory capacity is increased false alarms and number of fixations. A consequence of increased IU symptoms combined with low spatial working memory is the reduction in predictive search. The importance of this finding is considered further in the General Discussion. The pervasive influence of IU on behavioral and eye movement measures contrasts with the more limited influence of trait and state anxiety upon eye movements. Trait and state anxiety did not have any main effects upon eye movements.

\section{General Discussion}

Previous literature on visual search has largely been restricted to searching for targets in static displays. This study aimed to extend this to explore target detection in dynamically changing color displays. The aims were twofold: (1) to examine evidence for predictive monitoring in dynamically changing color displays for the onset of targets and (2) to identify a set of individual factors that support effective monitoring and target detection.

The results of Experiments 1 - 3 directly addressed the first aim. Target detection was no more accurate or faster when displays changed according to an ordered scale compared to when changes were randomized, but was least accurate and slowest in the shuffled condition of Experiment 2. In all experiments, items that changed according to a psychologically ordered color scale led to fixations being made to target-similar distractors that were potentially predictive of target onsets (see also Donnelly et al., 2006). These data were consistent with participants using a broad template to help locate possible targets, however, this process was error prone and false alarms were made. Further, while the use of prediction was associated with specific types of error, it never had a negative impact on the overall speed or accuracy of 
target detection and the results of Experiment 1 suggest that it allowed more forthcoming targets to be identified.

Contrasting the randomized condition of Experiment 1 with the shuffled condition of Experiment 2 revealed a second important result. The presence of arbitrarily defined structure within the color changes interfered with target detection more than randomized changes. This result has significant implications for how dynamically changing data should be visualized. Given that the pseudo-coloring of images cannot be done on random grounds, color scales must translate whatever property is being imaged meaningfully to the properties of psychological color space. Scales that are ordered in a way that does not conform to psychological color space will likely lead to misses.

These suggestions fit with two related principles of display design, the semantic mapping principle and the proximity compatibility principle. The semantic mapping principle states that performance when interacting with a display depends upon the quality of the mapping between the domain (or data) and the display (Bennett \& Flach, 1992, 2011). The proximity compatibility principle states that performance will be improved if information that should be processed together is integrated in some way within the display (Wickens, Gordon, Liu, \& Lee, 1998; Wickens, Hollands, Banbury, \& Parasuraman, 2015). In the context of the present task, both of these principles are compatible with our findings that performance suffered when color scales did not incorporate a meaningful order.

The explanation we have offered for the raised false alarm rate when first fixating distractors as TPDs in the ordered condition is that they are first identified using a broad template. Having been prioritized for monitoring, target detection requires a second match to a more tightly specified template and it is this second match that leads to false alarms.

Experiment 3 highlighted a set of individual characteristics that can be used to identify those most likely to make false alarms, specifically the combination of high IU and low verbal WMC. In other words, we can predict those most likely to produce monitoring errors when 
responding to target onsets. There is face validity to why low WMC and IU might lead to increased false alarm rates. Holding back 'target' responses when items are similar to targets require maintenance of broad and specific target templates, judgment and patience. Reduced capability to sustain templates, allied to discomfort in maintaining ambiguity lead to a tendency to make affirmative 'target' decisions. The roles that WMC and IU have in determining eye movement in the predictive monitoring task reported here is reminiscent of other recently reported studies indicating that associations between performance in cognitive tasks with symptoms of anxiety is most evident for individuals with low attentional control (Berggren \& Derakshan, 2013). Moreover, the present findings are consistent with a search strategy reflecting a hypervigilant broadening of attention in individuals with low IU and low WMC (review by Richards et al, 2014). A practical outcome of this finding is that an assessment of IU and WMC might be particularly beneficial in selecting personnel for scenarios where the cost associated with a false alarm to a color target onset is critical (for example, when monitoring visual displays for threat in battlefield scenarios). It is interesting that this interaction involved verbal WMC in the context of a task with no obvious verbal element. However, there is evidence of verbal WM being utilized in non-verbal tasks (Anderson, Mannan, Rees, Sumner, \& Kennard, 2008; Soto \& Humphreys, 2007). We suggest that participants used verbal labels to aid their discrimination of similar colors, especially those surrounding the target.

Experiment 3 found no evidence that target prevalence influenced the manner of predictive monitoring or overall hit rate. However, when considered in terms of the total time for which targets were present, both the high and low prevalence conditions of Experiment 3 involved relatively low target prevalence levels. This suggests that, in dynamic tasks, it is important to consider target prevalence not only in terms of the percentage of target-present trials, but also in terms of the percentage of the total display time for which targets are present. It is possible that in conditions of higher target prevalence, and the increased cue validity of 
TPDs that this would entail, predictive detection might be associated with a reliable benefit to performance. The use of a liberal criterion to identify targets in low prevalence search suggests that target prevalence may act differently in the present paradigm, compared to how it influences search in more traditional visual search tasks where reducing prevalence is associated with the adoption of increasingly conservative response criteria (Wolfe \& Van Wert, 2010). While in need of further study, these data suggest a need to consider how the prevalence effect generalizes beyond simple visual search.

\section{Conclusions and Implications}

The present study examined target detection within dynamically changing visual displays. When displays changed according to a psychologically ordered color space, targetpredictive distractors located one color step either side of the target color were prioritized and monitored. While the ability to predict and monitor potential targets was not associated with any improvements in overall task performance compared to randomized displays, displays that changed according to a shuffled color space interfered greatly with performance. We conclude that participants use a broad attentional template to prioritize distractors for monitoring when stimulus conditions allow and that the use of a broad template allows more forthcoming targets to be identified. However, an inevitable risk of using a broad attentional template in the context of a psychologically ordered color space is an elevated false alarm rate to target-similar stimuli. The results of Experiment 3 further suggest that individuals who are high in intolerance of uncertainty and low in verbal working memory capacity are particularly susceptible to making false alarms. The results of Experiments $1-3$ have clear implications for how we use color to represent dynamically changing data values. Principally, when choosing how to use color to represent dynamically changing data, a psychologically ordered color scale will allow those monitoring to predict likely target onsets. Incremental changes on such a scale will also maintain the continuity of color change, avoiding abrupt, highly salient, color changes that can 
interfere with target detection. That said, there are some for whom the use of an ordered color scale comes with a risk, specifically, individuals with high IU and low verbal WMC. The increased false alarm rates for these individuals suggest they may not be suited to such monitoring tasks, especially if there are significant risks or costs associated with pre-emptive target responses, such as in the military domain. These attributes are quick and easy to assess and could be used to test individual aptitude for monitoring dynamic visual displays. Beyond these individual characteristics, it is likely that the costs to predictive monitoring will have the greatest impact on tasks where targets are rare. In these circumstances, it is possible that these costs could be partially mitigated by artificially increasing target prevalence, such as in threat image projection in airport security screening (Godwin et al., 2010). In summary, the current study has pointed to issues of concern in how best to represent dynamically changing color information to allow target detection and the predictive monitoring for target onsets, and who might perform these types of task well. 


\section{References}

Anderson, E. J., Mannan, S., Rees, G., Sumner, P., \& Kennard, C. (2008). A role for spatial and nonspatial working memory processes in visual search. Experimental Psychology, 55(5), 301-312. http://doi.org/10.1027/1618-3169.55.5.301

Bach, M. (1996). The Freiburg Visual Acuity test--automatic measurement of visual acuity. Optometry and Vision Science, 73(1), 49-53.

Bates, D., Mächler, M., Bolker, B., \& Walker, S. (2014). Fitting Linear Mixed-Effects Models using lme4. Journal of Statistical Software, 67(1), 51. http://doi.org/10.18637/jss.v067.i01

Bennett, K. B., \& Flach, J. M. (1992). Graphical Displays: Implications for Divided Attention, Focused Attention, and Problem Solving. Human Factors: The Journal of the Human Factors and Ergonomics Society, 34(5), 513-533.

http://doi.org/10.1177/001872089203400502

Bennett, K. B., \& Flach, J. M. (2011). Display and Interface Design: Subtle Science, Exact Art (1st ed.). Boca Raton, FL, USA: CRC Press, Inc.

Berggren, N., \& Derakshan, N. (2013). Attentional control deficits in trait anxiety: why you see them and why you don't. Biological Psychology, 92(3), 440-6.

http://doi.org/10.1016/j.biopsycho.2012.03.007

Birrell, J., Meares, K., Wilkinson, A., \& Freeston, M. (2011). Clinical Psychology Review Toward a definition of intolerance of uncertainty : A review of factor analytical studies of the Intolerance of Uncertainty Scale. Clinical Psychology Review, 31(7), 1198-208. http://doi.org/10.1016/j.cpr.2011.07.009

Bleckley, M. K., Durso, F. T., Crutchfield, J. M., Engle, R. W., \& Khanna, M. M. (2003). Individual differences in working memory capacity predict visual attention allocation. Psychonomic Bulletin \& Review, 10(4), 884-889. http://doi.org/10.3758/BF03196548

Caggiano, D. M., \& Parasuraman, R. (2004). The role of memory representation in the vigilance decrement. Psychonomic Bulletin \& Review, 11(5), 932-937. 
http://doi.org/10.3758/BF03196724

Carleton, N. R., Norton, M. A. P. J., \& Asmundson, G. J. G. (2007). Fearing the unknown: a short version of the Intolerance of Uncertainty Scale. Journal of Anxiety Disorders, 21(1), 105-17. http://doi.org/10.1016/j.janxdis.2006.03.014

Derakshan, N., \& Eysenck, M. W. (2009). Anxiety, Processing Efficiency, and Cognitive Performance. European Psychologist, 14(2), 168-176. http://doi.org/10.1027/10169040.14 .2 .168

Derakshan, N., Smyth, S., \& Eysenck, M. W. (2009). Effects of state anxiety on performance using a task-switching paradigm: An investigation of attentional control theory. Psychonomic Bulletin \& Review, 16(6), 1112-1117. http://doi.org/10.3758/PBR.16.6.1112

Donnelly, N., Cave, K. R., Welland, M., \& Menneer, T. (2006). Breast screening, chicken sexing and the search for oil: challenges for visual cognition. Geological Society, London, Special Publications, 254(1), 43-55. http://doi.org/10.1144/gsl.sp.2006.254.01.04

Drew, T., Võ, M. L.-H., \& Wolfe, J. M. (2013). The Invisible Gorilla Strikes Again: Sustained Inattentional Blindness in Expert Observers. Psychological Science, 24(9), 1848-1853. http://doi.org/10.1177/0956797613479386

Eysenck, M. W., \& Derakshan, N. (2011). New perspectives in attentional control theory. Personality and Individual Differences, 50(7), 955-960. http://doi.org/10.1016/j.paid.2010.08.019

Eysenck, M. W., Derakshan, N., Santos, R., \& Calvo, M. G. (2007). Anxiety and cognitive performance: attentional control theory. Emotion (Washington, D.C.), 7(2), 336-53. http://doi.org/10.1037/1528-3542.7.2.336

Fergus, T. A., Bardeen, J. R., \& Wu, K. D. (2013). Intolerance of Uncertainty and UncertaintyRelated Attentional Biases: Evidence of Facilitated Engagement or Disengagement Difficulty? Cognitive Therapy and Research, 37(4), 735-741. 
http://doi.org/10.1007/s10608-012-9509-9

Fergus, T. A., \& Carleton, N. R. (2016). Intolerance of uncertainty and attentional networks: Unique associations with alerting. Journal of Anxiety Disorders, 41, 59-64. http://doi.org/10.1016/j.janxdis.2016.03.010

Fletcher, R. (1998). The City University Colour Vision Test (3rd ed.). London: Keeler.

Fukuda, K., \& Vogel, E. K. (2011). Individual differences in recovery time from attentional capture. Psychological Science, 22(3), 361-368.

http://doi.org/10.1177/0956797611398493

Godwin, H. J., Menneer, T., Cave, K. R., Helman, S., Way, R. L., \& Donnelly, N. (2010). The impact of Relative Prevalence on dual-target search for threat items from airport X-ray screening. Acta Psychologica, 134(1), 79-84. http://doi.org/10.1016/j.actpsy.2009.12.009

Godwin, H. J., Menneer, T., Riggs, C. a, Cave, K. R., \& Donnelly, N. (2014). Perceptual failures in the selection and identification of low-prevalence targets in relative prevalence visual search. Attention, Perception \& Psychophysics. http://doi.org/10.3758/s13414-014$0762-8$

Hout, M. C., \& Goldinger, S. D. (2015). Target templates: the precision of mental representations affects attentional guidance and decision-making in visual search. Attention, Perception, \& Psychophysics, 77(1), 128-149. http://doi.org/10.1016/j.surg.2006.10.010.Use

Jardine, N. L., \& Moore, C. M. (2015). Losing the Trees for the Forest in Dynamic Visual Search. Journal of Experimental Psychology: Human Perception and Performance, 42(5), 617-630. http://doi.org/10.1037/xhp0000186

Kane, M. J., Bleckley, M. K., Conway, A. R. A., \& Engle, R. W. (2001). A ControlledAttention View of Working-Memory Capacity. Journal of Experimental Psychology: General, 130, 169-183. http://doi.org/10.1037/0096-3445.130.2.169

Klein, R. M. (2000). Inhibition of return. Trends in Cognitive Sciences, 4(4), 138-147. 
http://doi.org/10.1016/S1364-6613(00)01452-2

Kunar, M. a, \& Watson, D. G. (2011). Visual search in a multi-element asynchronous dynamic (MAD) world. Journal of Experimental Psychology. Human Perception and Performance, 37(4), 1017-1031. http://doi.org/10.1037/a0023093

Kunar, M. a, \& Watson, D. G. (2014). When are abrupt onsets found efficiently in complex visual search? Evidence from multielement asynchronous dynamic search. Journal of Experimental Psychology. Human Perception and Performance, 40(1), 232-52. http://doi.org/10.1037/a0033544

Lam, R. W., Michalak, E. E., \& Swinson, R. P. (2005). Assessment scales in depression, mania, and anxiety. Clinical Neuroscience, 198. http://doi.org/10.1192/pb.30.10.398

Mcvay, J. C., \& Kane, M. J. (2012). Why Does Working Memory Capacity Predict Variation in Reading Comprehension? On the Influence of Mind Wandering and Executive Attention. Journal of Experimental Psychology: General, 141(2), 302-320. http://doi.org/10.1037/a0025250.Why

Owens, M., Stevenson, J., Hadwin, J. A., \& Norgate, R. (2014). When does anxiety help or hinder cognitive test performance? The role of working memory capacity. British Journal of Psychology, 105(1), 92-101. http://doi.org/10.1111/bjop.12009

Pacheco-Unguetti, A. P., Acosta, A., Lupiáñez, J., Román, N., \& Derakshan, N. (2012). Response inhibition and attentional control in anxiety. The Quarterly Journal of Experimental Psychology, 65(June 2014), 646-660. http://doi.org/10.1080/17470218.2011.637114

Peltier, C., \& Becker, M. W. (2017). Individual differences predict low prevalence visual search performance. Cognitive Research: Principles and Implications. http://doi.org/10.1186/s41235-016-0042-3

Richards, H. J., Benson, V., Donnelly, N., \& Hadwin, J. A. (2014). Exploring the function of selective attention and hypervigilance for threat in anxiety. Clinical Psychology Review, 
34(1), 1-13. http://doi.org/10.1016/j.cpr.2013.10.006

Schwark, J. D., Sandry, J., Macdonald, J., \& Dolgov, I. (2012). False feedback increases detection of low-prevalence targets in visual search. Attention, Perception \& Psychophysics, 74(8), 1583-9. http://doi.org/10.3758/s13414-012-0354-4

Shackman, A. J., Sarinopoulos, I., Maxwell, J. S., Pizzagalli, D. A., Lavric, A., \& Davidson, R. J. (2006). Anxiety selectively disrupts visuospatial working memory. Emotion (Washington, D.C.), 6, 40-61. http://doi.org/10.1037/1528-3542.6.1.40

Sörqvist, P., \& Marsh, J. E. (2015). How Concentration Shields Against Distraction. Current Directions in Psychological Science, 24(4), 267-272. http://doi.org/10.1177/0963721415577356

Soto, D., \& Humphreys, G. W. (2007). Automatic guidance of visual attention from verbal working memory. Journal of Experimental Psychology. Human Perception and Performance, 33(3), 730-737. http://doi.org/10.1037/0096-1523.33.3.730

Spielberger, C. D., Gorsuch, R. L., Lushene, P. R., Vagg, P. R., \& Jacobs, A. G. (1983). Manual for the State-Trait Anxiety Inventory (Form Y). Manual for the statetrait anxiety inventory STAI.

Stroud, M. J., Menneer, T., Cave, K. R., \& Donnelly, N. (2012). Using the dual-target cost to explore the nature of search target representations. Journal of Experimental Psychology: Human Perception and Performance, 38(1), 113-122. http://doi.org/10.1037/a0025887

Stroud, M. J., Menneer, T., Cave, K. R., Donnelly, N., \& Rayner, K. (2011). Search for multiple targets of different colours: misguided eye movements reveal a reduction of colour selectivity. Applied Cognitive Psychology, 25(6), 971-982.

Szalma, J. L. (2009). Individual differences: incorporating human variation into human factors/ergonomics research and practice. Theoretical Issues in Ergonomics Science, 10(January 2015), 377-379. http://doi.org/10.1080/14639220903106361

Vickers, D., \& Leary, J. N. (1983). Criterion Control in Signal Detection. Human Factors: The 
Journal of the Human Factors and Ergonomics Society, 25(3), 283-296.

Warm, J. S., Finomore, V. S., Vidulich, M. A., \& Funke, M. E. (2015). Vigilance: A Perceptual Challenge. In R. R. Hoffman, P. A. Hancock, M. W. Scerbo, R. Parasuraman, \& J. L. Szalma (Eds.), The Cambridge Handbook of Applied Perception Research (pp. 241-283). Cambridge, UK: Cambridge University Press. http://doi.org/10.1017/CBO9780511973017.018

Warm, J. S., \& Parasuraman, R. (2008). Vigilance Requires Hard Mental Work and Is Stressful. Human Factors, 50(3), 433-441. http://doi.org/10.1518/001872008X312152.

Wickens, C. D., Gordon, S. E., Liu, Y., \& Lee, J. (1998). An introduction to human factors engineering.

Wickens, C. D., Hollands, J. G., Banbury, S., \& Parasuraman, R. (2015). Engineering psychology \& human performance. Psychology Press.

Wolfe, J. M., Horowitz, T. S., Wert, M. J. Van, Kenner, N. M., Place, S. S., \& Kibbi, N. (2007). Low target prevalence is a stubborn source of errors in visual search tasks. Journal of Experimental Psychology General, 136(4), 623-638. http://doi.org/10.1037/0096-3445.136.4.623.

Wolfe, J. M., \& Van Wert, M. J. (2010). Varying target prevalence reveals two dissociable decision criteria in visual search. Current Biology, 20(2), 121-4. http://doi.org/10.1016/j.cub.2009.11.066 
Table 1

Mean Hit Rate, Response Time (RT) and False Alarm Rate in Experiments 1, 2 and 3

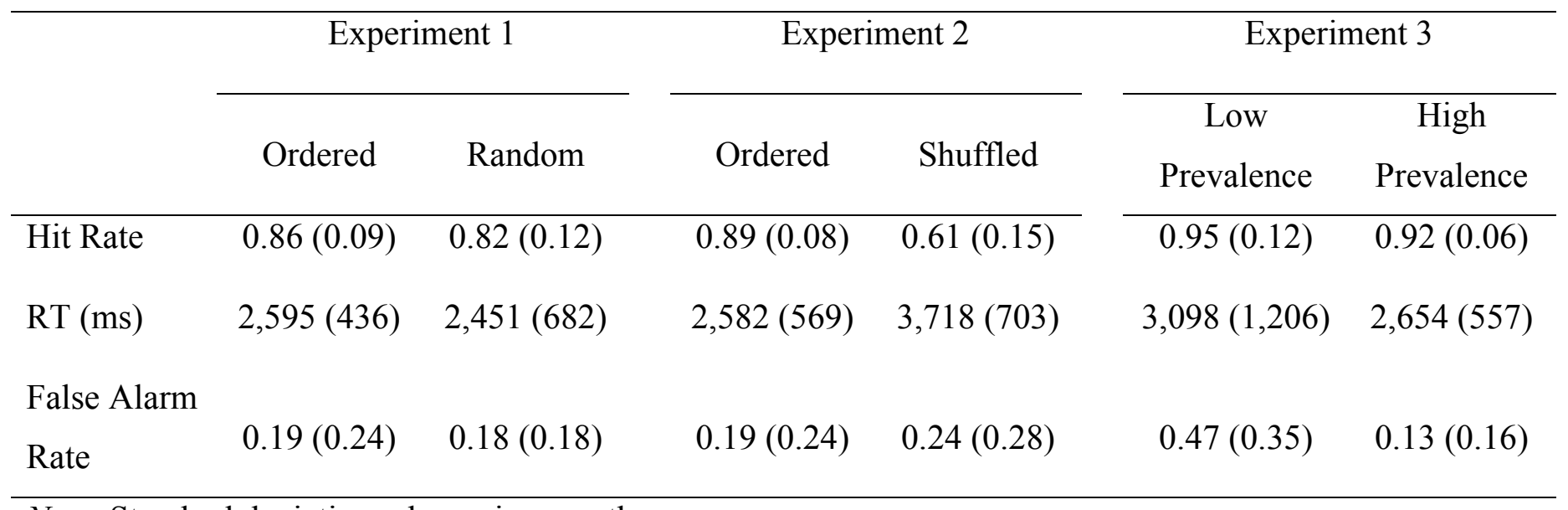

Note. Standard deviations shown in parentheses 
Table 2.

GLMMs on the effects of target prevalence, working memory capacity (WMC), anxiety and intolerance of uncertainty (IU) upon task performance in terms of hit rate, false alarm rate and color steps (limited to 0 or 1) from target color at first fixation and LMMs for response time (RT) and eye movement measures of trial total scanpath length, trial total number of fixations and fixation duration. Standard errors of estimates are shown in parentheses and dashes indicate that a factor was not included in a particular model.

\begin{tabular}{|c|c|c|c|c|c|c|c|}
\hline & Hit Rate & $\begin{array}{l}\text { False Alarm } \\
\text { Rate }\end{array}$ & $\mathrm{RT}$ & $\begin{array}{l}\text { Color Step At } \\
\text { First Fixation }\end{array}$ & $\begin{array}{l}\text { Trial Scanpath } \\
\text { Length }\end{array}$ & $\begin{array}{l}\text { Trial Number } \\
\text { Of Fixations }\end{array}$ & $\begin{array}{l}\text { Fixation } \\
\text { Duration }\end{array}$ \\
\hline Constant & $\begin{array}{l}2.53^{*} \\
(0.15)\end{array}$ & $\begin{array}{c}0.37 \\
(0.23)\end{array}$ & $\begin{array}{l}7.73^{*} \\
(0.04)\end{array}$ & $\begin{array}{l}-0.17 \\
(0.12)\end{array}$ & $\begin{array}{l}630.17^{*} \\
(31.98)\end{array}$ & $\begin{array}{c}114.01^{*} \\
(4.65)\end{array}$ & $\begin{array}{l}5.55^{*} \\
(0.03)\end{array}$ \\
\hline $\begin{array}{l}\text { Target } \\
\text { Prevalence }\end{array}$ & - & $\begin{array}{c}-2.64^{*} \\
(0.14)\end{array}$ & - & - & $\begin{array}{l}61.60^{*} \\
(1.10)\end{array}$ & $\begin{array}{l}3.89^{*} \\
(1.19)\end{array}$ & - \\
\hline Verbal WMC & $\begin{array}{c}2.66 \\
(1.43)\end{array}$ & $\begin{array}{l}-2.17 \\
(1.95)\end{array}$ & $\begin{array}{l}-0.39 \\
(0.34)\end{array}$ & $\begin{array}{l}-1.24 \\
(1.26)\end{array}$ & $\begin{array}{c}340.86 \\
(287.67)\end{array}$ & $\begin{array}{c}60.19 \\
(46.01)\end{array}$ & $\begin{array}{l}-0.18 \\
(0.28)\end{array}$ \\
\hline Spatial WMC & - & - & - & $\begin{array}{c}0.85 \\
(1.00)\end{array}$ & - & $\begin{array}{l}-54.67 \\
(41.38)\end{array}$ & - \\
\hline $\mathrm{IU}$ & $\begin{array}{l}-0.01 \\
(0.02)\end{array}$ & $\begin{array}{l}0.07^{*} \\
(0.02)\end{array}$ & $\begin{array}{c}0.002 \\
(0.004)\end{array}$ & $\begin{array}{c}-0.002 \\
(0.01)\end{array}$ & $\begin{array}{l}-0.10 \\
(3.51)\end{array}$ & $\begin{array}{c}0.21 \\
(0.46)\end{array}$ & $\begin{array}{l}-0.002 \\
(0.003)\end{array}$ \\
\hline Trait Anxiety & - & - & - & - & $\begin{array}{c}1.31 \\
(3.56)\end{array}$ & - & - \\
\hline $\begin{array}{l}\text { Target Prevalence } \mathrm{x} \\
\text { IU }\end{array}$ & - & $\begin{array}{c}-0.03^{*} \\
(0.01)\end{array}$ & - & - & - & - & - \\
\hline $\begin{array}{l}\text { Target Prevalence x } \\
\text { Trait Anxiety }\end{array}$ & - & - & - & - & $\begin{array}{l}2.97^{*} \\
(0.97)\end{array}$ & & - \\
\hline $\begin{array}{l}\text { Verbal WMC x } \\
\text { IU }\end{array}$ & & $\begin{array}{c}-0.41^{*} \\
(0.17)\end{array}$ & - & - & - & $\begin{array}{c}-8.60^{*} \\
(4.08)\end{array}$ & $\begin{array}{c}0.05 \\
(0.03)\end{array}$ \\
\hline $\begin{array}{l}\text { Spatial WMC x } \\
\text { IU }\end{array}$ & - & - & - & $\begin{array}{c}-0.33^{*} \\
(0.12)\end{array}$ & - & - & - \\
\hline Observations & 1,988 & 2,657 & 1,829 & 1,783 & 1,448 & 1,448 & 1,448 \\
\hline Log Likelihood & -533.42 & $-1,022.08$ & $-1,686.57$ & $-1,191.72$ & $-8,704.56$ & $-5,599.90$ & $1,613.21$ \\
\hline Akaike Inf. Crit. & $1,074.84$ & $2,058.17$ & $3,383.15$ & $2,395.45$ & $17,425.12$ & $11,215.79$ & $-3,214.41$ \\
\hline Bayesian Inf. Crit. & $1,097.22$ & $2,099.36$ & $3,410.71$ & $2,428.36$ & $17,467.34$ & $11,225.02$ & $-3,182.74$ \\
\hline
\end{tabular}




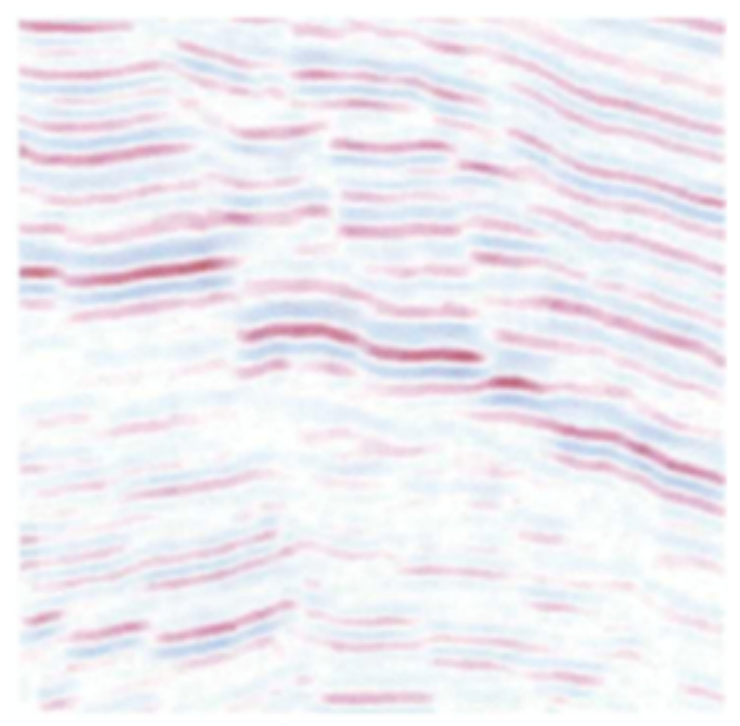

Figure 1. Example of a 2D slice taken from a 3D data volume. 


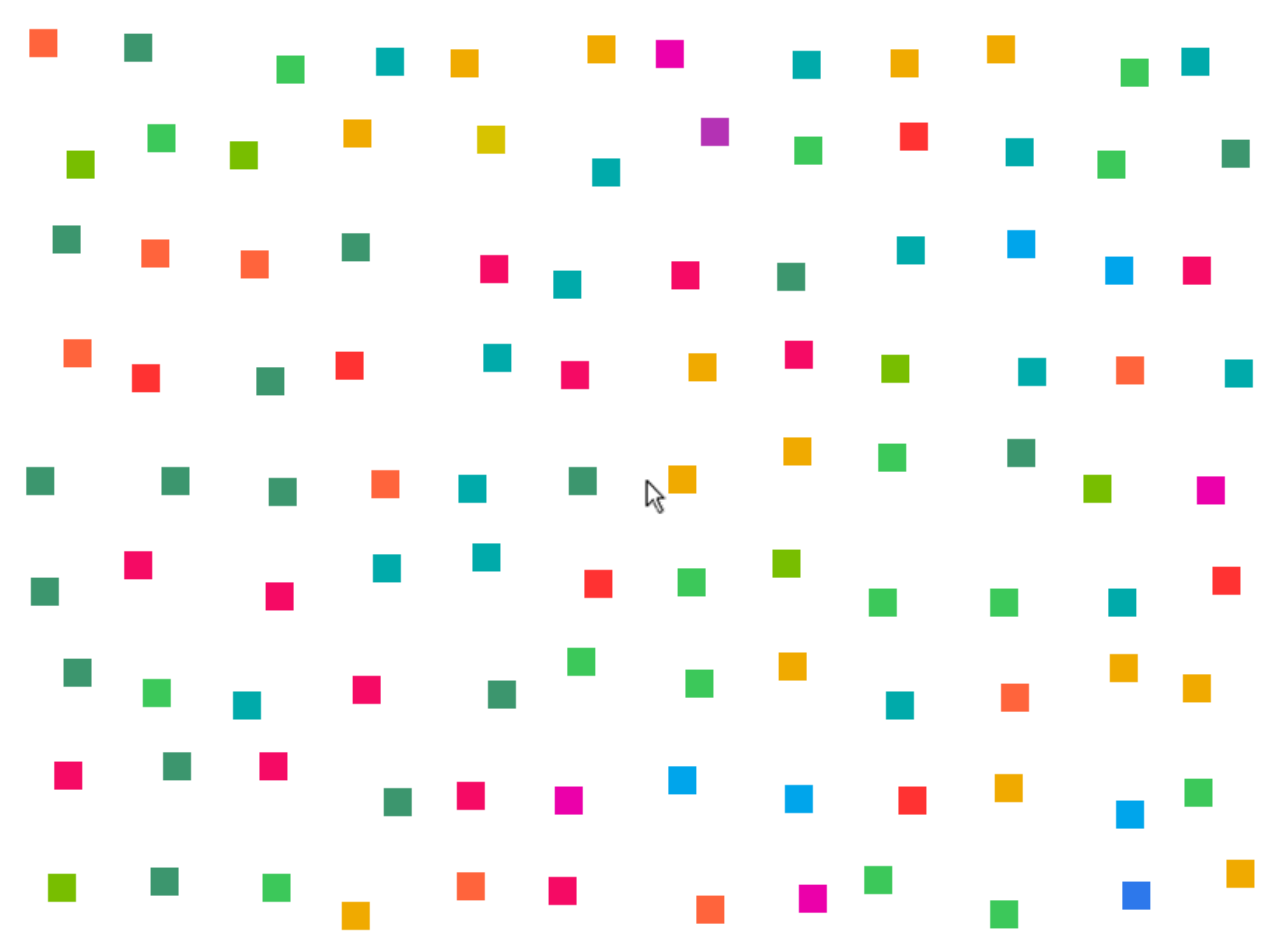

Figure 2. Sample stimulus display 

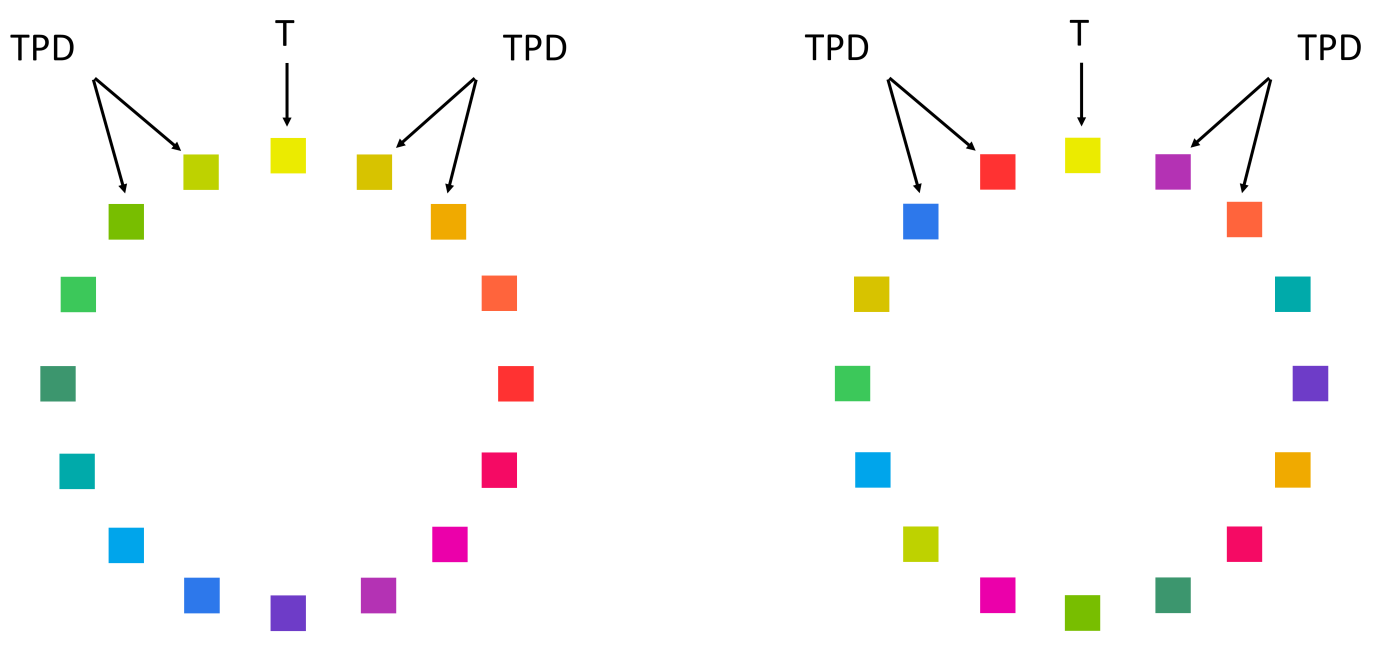

Figure 3. Ordered color scale (left) and an example of a shuffled color scale (right; used in Experiment 2 only) showing example of what were classed target-predictive distractors (TPDs) when the target $(\mathrm{T})$ was the indicated color. 


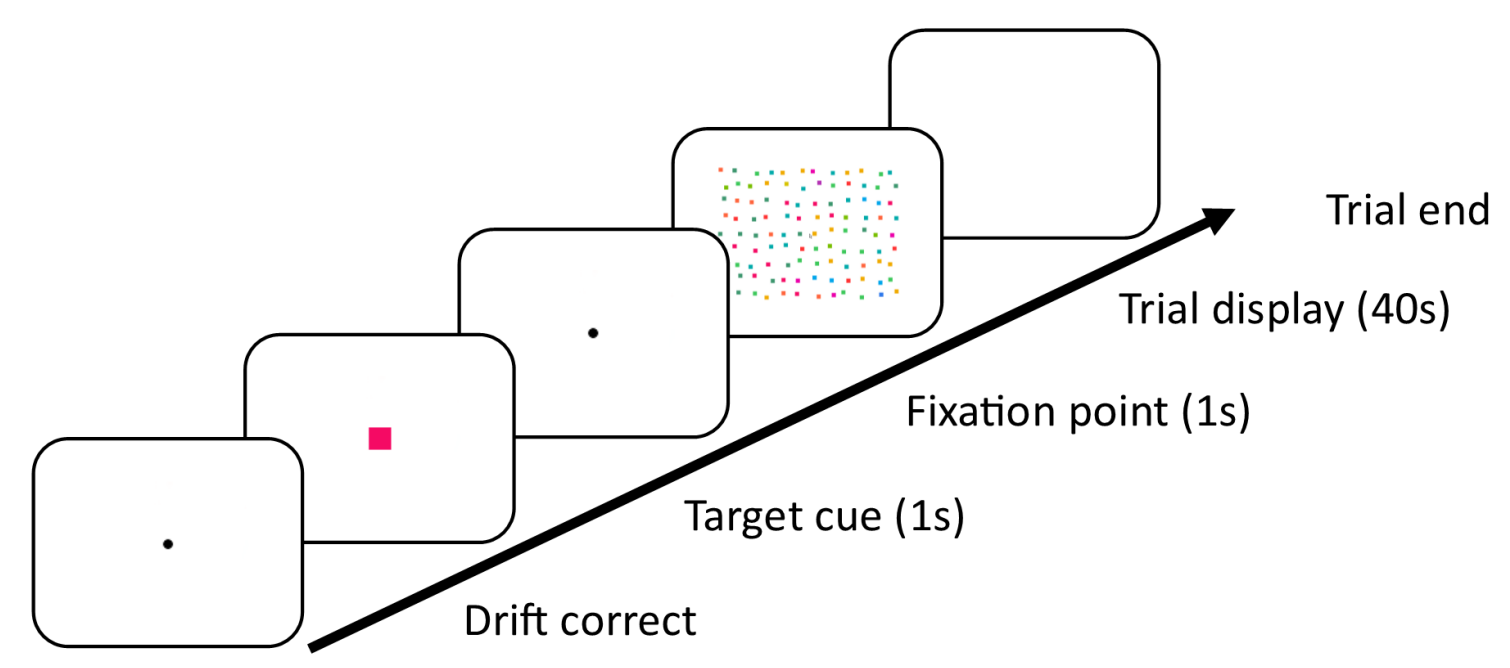

Figure 4. Trial sequence 

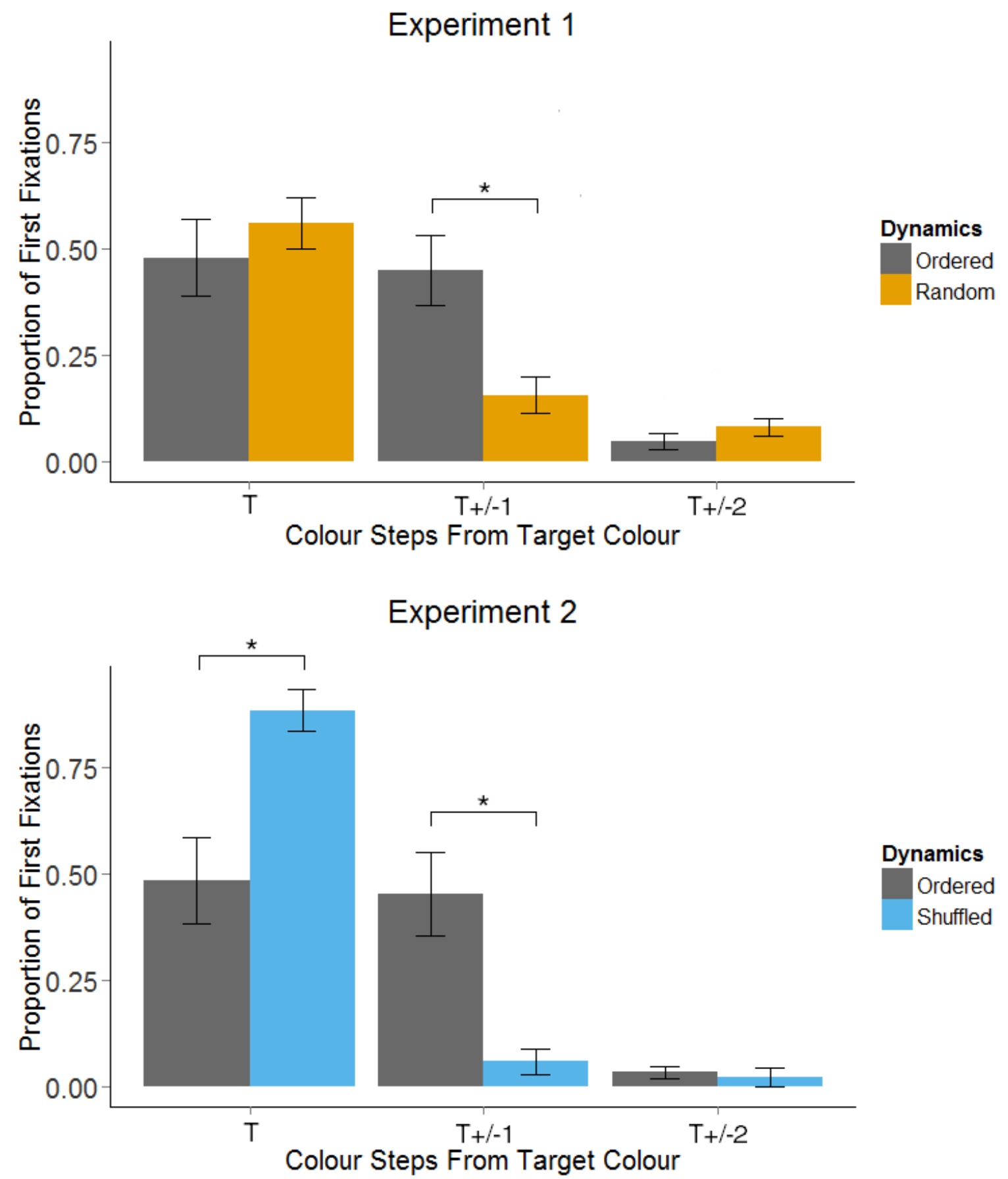

Figure 5. The proportion of first fixations to active targets and forthcoming targets as a function of the number of color steps from the target color under ordered sequential, randomized and shuffled display dynamics in Experiments 1 (top) and 2 (bottom). Error bars show 95\% CIs and ' $*$ ' indicates a pairwise comparison where $p<.017$. 

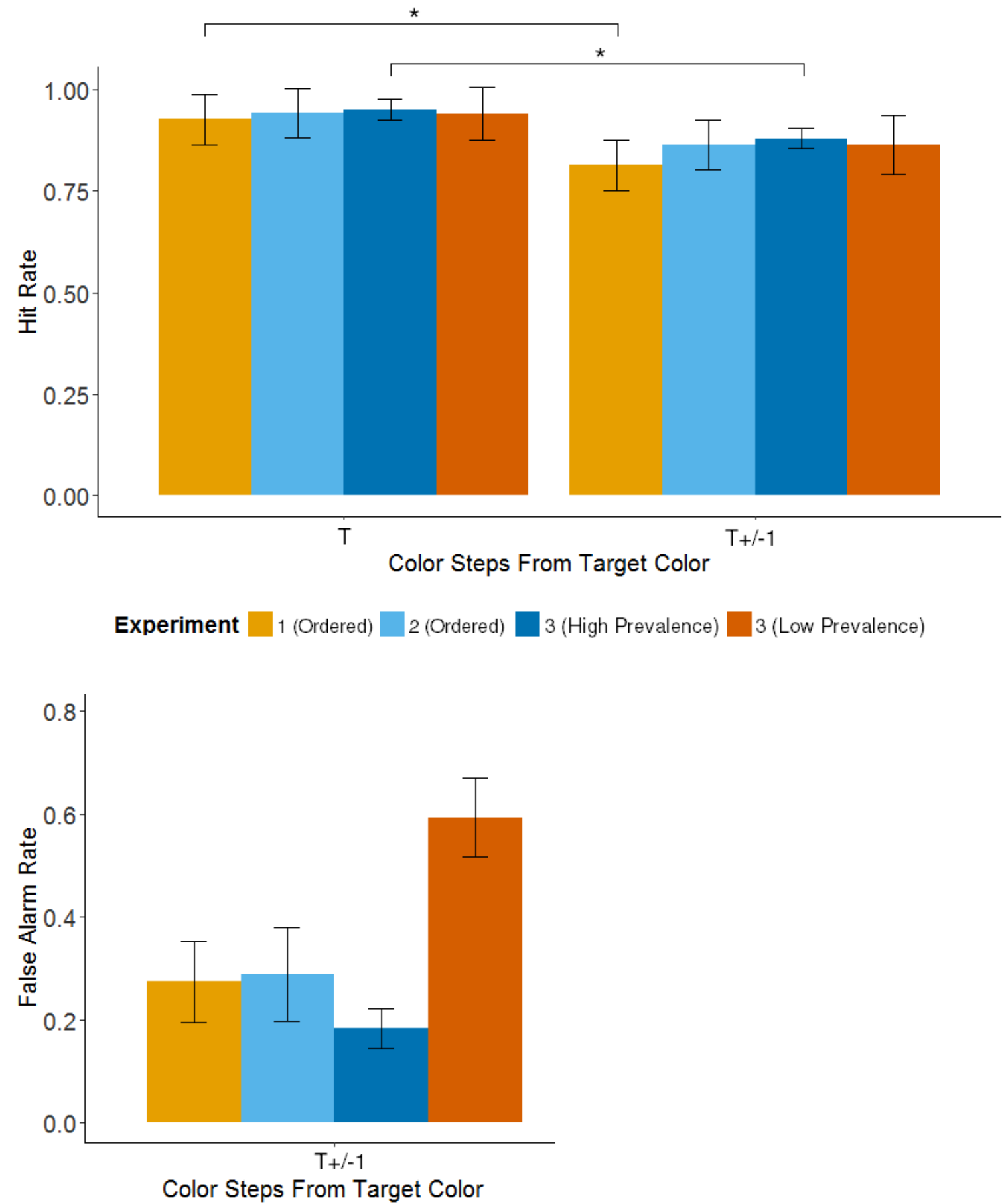

Figure 6. The hit rates for targets first fixated as targets and as target-predictive distractors at $\mathrm{T}+/-1$ color step (top) and the false alarm rates for forthcoming targets first fixated as targetpredictive distractors at $\mathrm{T}+/-1$ color step (bottom) across the ordered conditions in Experiments 1 and 2, and both prevalence levels in Experiment 3. Error bars show 95\% CIs and '*' indicates a pairwise comparison where $p<.05$. 


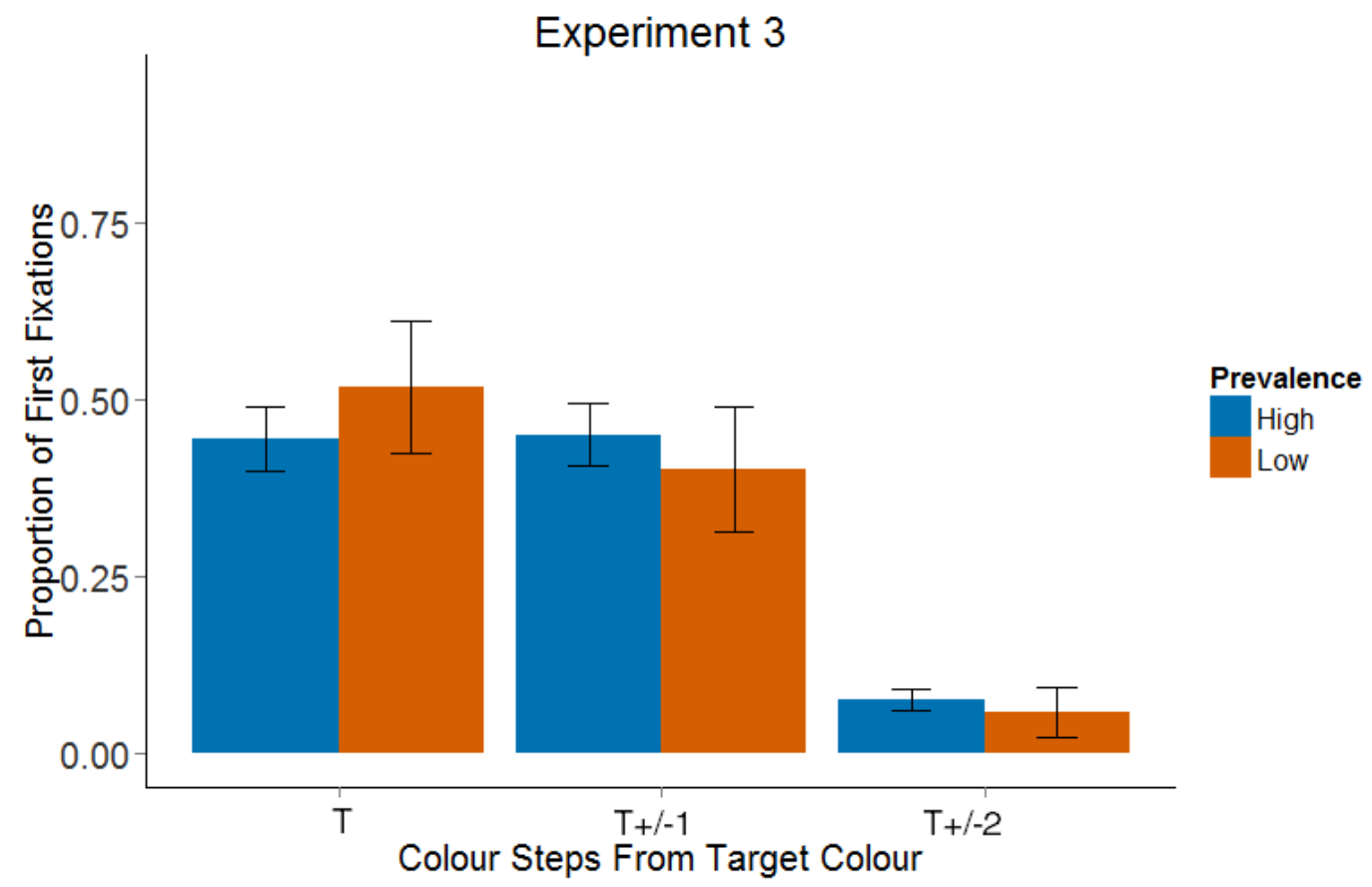

Figure 7. The proportion of first fixations to targets and forthcoming targets as a function of the number of color steps from the target color under high and low target prevalence in Experiment 3 (error bars show 95\% CIs). 

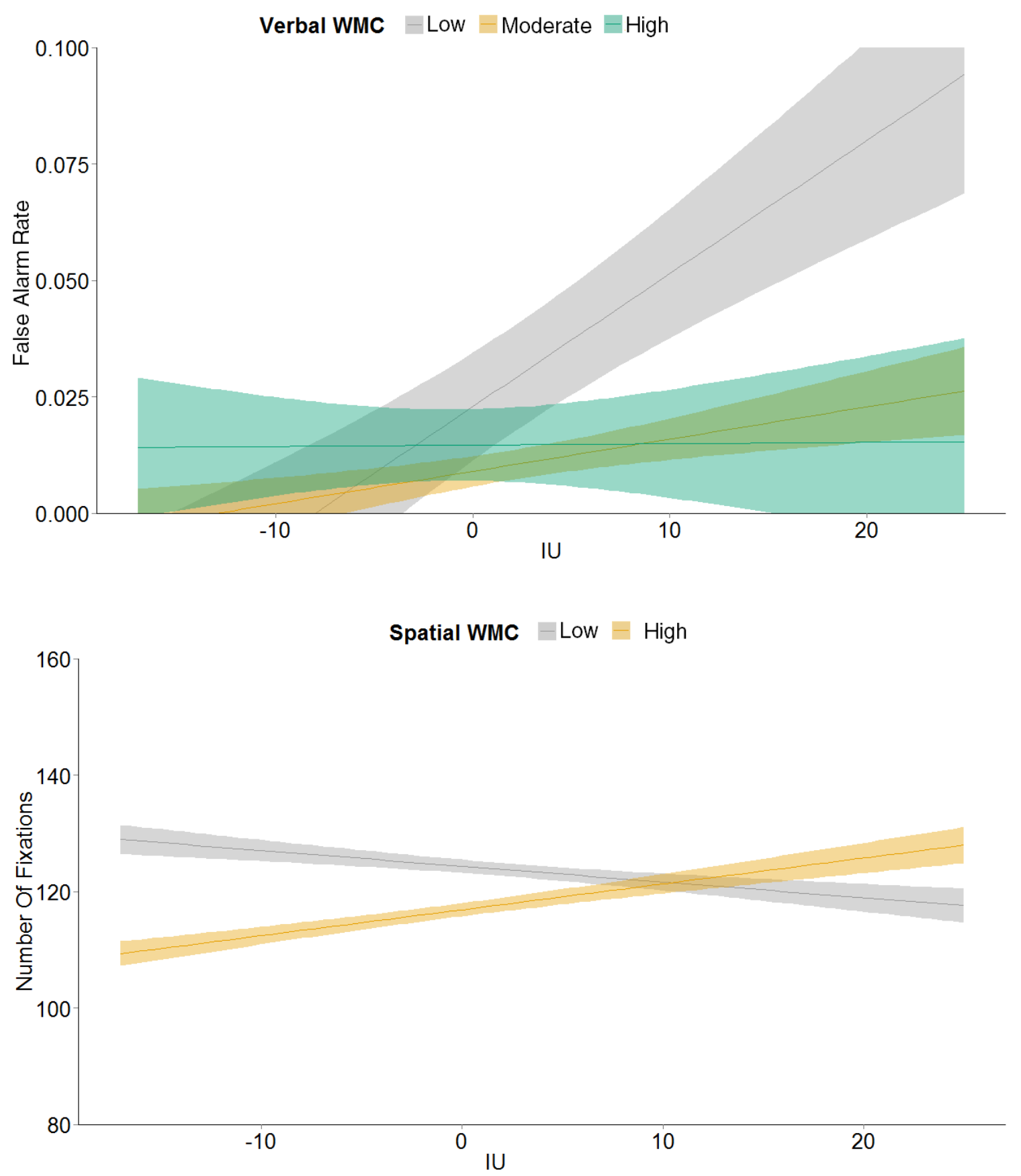

Figure 8. False alarm rate and intolerance of uncertainty (IU) at low, moderate and high levels of verbal working memory capacity (WMC; top) and number of fixations and IU at low and high levels of spatial WMC (bottom; shaded regions show continuous 95\% CIs). Verbal WMC was split at the $33^{\text {rd }}$ and $66^{\text {th }}$ percentiles to give three categories and spatial WMC was split at the median to give two categories. This categorization is only for the purposes of visualization and these data were included in all analyses as continuous data. 